\title{
Multiple myeloma cells alter the senescence phenotype of bone marrow mesenchymal stromal cells under participation of the DLK1-DIO3 genomic region
}

Rimma Berenstein ${ }^{1 *}$, Olga Blau ${ }^{1}$, Axel Nogai ${ }^{1}$, Marlies Waechter ${ }^{1}$, Ekaterina Slonova ${ }^{1}$, Martin Schmidt-Hieber ${ }^{2}$, Annegret Kunitz', Antonio Pezzutto ${ }^{1}$, Bernd Doerken ${ }^{1}$ and Igor Wolfgang Blau ${ }^{1}$

\begin{abstract}
Background: Alterations and senescence in bone marrow mesenchymal stromal cells of multiple myeloma patients (MM-BMMSCs) have become an important research focus. However the role of senescence in the pathophysiology of MM is not clear.

Methods: Correlation between senescence, cell cycle and microRNA expression of MM-BMMSCs $(n=89)$ was analyzed. Gene expression analysis, copy number analysis and methylation specific PCR were performed by Real-Time PCR. Furthermore, cyclin E1, cyclin D1, p16 and p21 genes were analyzed at the protein level using ELISA. Cell cycle and senescence were analyzed by FACS. MiRNA transfection was performed with miR-485-5p inhibitor and mimic followed by downstream analysis of senescence and cell cycle characteristics of MM-BMMSCs. Results were analyzed by Mann-Whitney $U$ test, Wilcoxon signed-rank test and paired t-test depending on the experimental set up.

Results: MM-BMMSCs displayed increased senescence associated $\beta$-galactosidase activity (SA- $\beta$ GalA), cell cycle arrest in S phase and overexpression of microRNAs. The overexpressed microRNAs miR-485-5p and miR-519d are located on DLK1-DIO3 and C19MC, respectively. Analyses revealed copy number accumulation and hypomethylation of both clusters. KMS12-PE myeloma cells decreased SA- $\beta$ GalA and influenced cell cycle characteristics of MM-BMMSCs. MiR-485-5p was significantly decreased in co-cultured MM-BMMSCs in connection with an increased methylation of DLK1-DIO3. Modification of miR-485-5p levels using microRNA mimic or inhibitor altered senescence and cell cycle characteristics of MM-BMMSCs.
\end{abstract}

Conclusions: Here, we show for the first time that MM-BMMSCs have aberrant methylation and copy number of the DLK1-DIO3 and C19MC genomic region. Furthermore, this is the first study pointing that multiple myeloma cells in vitro reduce both the senescence phenotype of MM-BMMSCs and the expression of miR-223 and miR-485-5p. Thus, it is questionable whether senescence of MM-BMMSCs plays a pathological role in active multiple myeloma or is more important when cell interaction with myeloma cells is inhibited. Furthermore, we found that MiR-485-5p, which is located on the DLK1-DIO3 cluster, seems to participate in the regulation of senescence status and cell cycle characteristics of MM-BMMSCs. Thus, further exploration of the microRNAs of DLK1-DIO3 could provide further insights into the origin of the senescence state and its reversal in MM-BMMSCs.

Keywords: Multiple myeloma, Bone marrow stromal cells, Senescence, Cell cycle, DLK1-DIO3, miR-485-5p

\footnotetext{
* Correspondence: rimma.berenstein@charite.de

${ }^{1}$ Department of Hematology, Oncology and Tumourimmunology, Charité Universitätsmedizin Berlin, Hindenburgdamm 30, 12200 Berlin, Germany Full list of author information is available at the end of the article
} 


\section{Background}

Multiple Myeloma (MM) is a B-cell malignancy characterized by the accumulation of malignant plasma cells (PC) within the bone marrow (BM) and the strong interaction between several cellular compartments [1].

BMMSCs support MM cell growth through different direct and indirect factors leading to increased tumor support and possible generation of drug resistance [2-10].

Thus, the surrounding tumor microenvironment has become a focal point of MM research. Several studies have suggested the genesis of constitutive abnormalities in MM-BMMSCs through interactions with MM cells [11-14]. For instance development of a senescence-like state in BMMSCs and thereby a modulated secretory profile, worsened osteogenic differentiation potential and inhibition of the T-cell proliferation were reported $[13,15]$. Senescence is a cellular state associated with the loss of proliferative capacity and changes in the secretion of pro-inflammatory cytokines and growth factors [16]. Senescent BMMSCs display an increased senescenceassociated $\beta$-galactosidase activity (SA- $\beta$ GalA) and irregular cell morphology. Usually the cell cycle of senescent cells is arrested at the $\mathrm{G}_{1} / \mathrm{S}$-transition point in combination with the overexpression of different cell cycle inhibitors as p21 and p16. In spite of the aberrant growth characteristics senescent cells remain metabolically active and therefore the secretion of pro-inflammatory mediators could promote tumorigenesis in neighboring premalignant cells [17-19]. Although some reports describe constitutive changes in MM-BMMSCs, the molecular mechanisms and pathways that induce senescence-associated abnormalities are largely unknown. Furthermore it is not clear whether alterations of MM-BMMSCs are important for the interaction between stromal cells and MM cells or are more an attendant phenomenon.

Two imprinted clusters in the human genome might contribute to the generation of senescence and induction of cellular changes in MM-BMMSCs [20-23]. The DLK1-DIO3 imprinted domain is located on chromosome $14 \mathrm{q} 32.2$ and expresses 53 microRNAs, whereas the imprinted cluster C19MC is located on chromosome 19q13 and codes for 59 microRNAs [24-26]. Allelic expression of DLK1-DIO3 is controlled through methylation of a regulatory region (IG-DMR) located about $15 \mathrm{~kb}$ upstream of the cluster and the expression of C19MC correlates with the epigenetic modulation of a $\mathrm{CpG}$-rich region located about $17.6 \mathrm{~kb}$ upstream [26,27].

Up to now no data on the role of the senescent phenotype of MM-BMMSCs for the progression of MM are available. Previously, we have shown that MM-BMMSCs exhibit overexpression of distinct microRNAs and an increased senescence phenotype as compared to healthy donor BMMSCs [28]. To further address this point we analyzed in this study the correlation between senescence status, cell cycle characteristics and microRNA expression of MM-BMMSCs. We chose microRNAs, which were previously reported to be deregulated in MM cells and play a potential role in inflammation-induced cellular senescence [21,29-32]. We further addressed the question of whether interaction of MM-BMMSCs with MM cells could modulate the cell cycle and senescence-like state of MM-BMMSCs by altering the expression of microRNA molecules. In this context we wanted to analyze whether the premature senescence status of MM-BMMSCs could be a specific effect induced by MM cells for increased tumor support. MiR-485-5p, which is encoded by the DLK1-DIO3 cluster, was found to be a potential modulator of the cell cycle and senescence status of MMBMMSCs. Based on this observation we finally investigated the effect of miR-485-5p mimics or inhibitor on the cell cycle and SA- $\beta$ GalA of MM-BMMSCs.

\section{Methods}

\section{Patient and donor characteristics}

BM samples from 89 patients with multiple myeloma $[\mathrm{n}=54$ with $\mathrm{MM}$ at the time of diagnosis and $\mathrm{n}=35$ at relapse] were included in the study. All patients had a treatment indication. Written informed consent was obtained from all patients and healthy donors in accordance with the Declaration of Helsinki and the ethical guidelines of the Charite University School of Medicine, which approved this study (Votum No.: EA4/131/13). Twelve bone marrow aspirates were received from healthy donors (see Additional file 1: Table S1: Patients and Donor Characteristics).

\section{Isolation and cultivation of BMMSCs}

BMMSCs from patients with MM (MM-BMMSCs) or donors (HD-BMMSCs) were isolated by the classical adhesion method and cultivated as previously described [33-35]. Non-hematopoietic cell characteristics were identified by flow cytometry by absence of CD45 and CD34 and presence of CD105 and CD90 expression with antibodies from Miltenyi Biotec. Data was acquired and analyzed with a FACS Calibur Flow Cytometer (BD Biosciences) and Flowing Software (Cell Imaging Core). Purity of isolated BMMSCs ranged from $94 \%$ to $99.5 \%$ (see Additional file 2: Figure S1: Purity of isolated BMMSCs).

\section{Co-Cultures and transwell cultures of MM-BMMSCs}

For co-cultures $5 \times 10^{4}$ MM-BMMSCs were seeded in a 6 -well plate and incubated for $4 \mathrm{~h}$. Then $5 \times 10^{4} \mathrm{KMS12-}$ PE myeloma cells were added followed by incubation for $72 \mathrm{~h}$. After incubation KMS12-PE cells were removed by rigorous pipetting and detachment was confirmed by microscopy. In addition the absence of $\mathrm{CD} 138^{+}$ cells was checked with FACS during cell cycle analysis. MM-BMMSCs were washed twice with PBS and 
applied for downstream analysis. Co-cultured KMS12-PE myeloma cells were pelleted and re-suspended in TRIzol for downstream analysis.

For transwell cultures $(0.4 \mu \mathrm{M}$ pore size, Corning) $2 \times 10^{4}$ MM-BMMSCs were seeded in the lower chamber of a 12-well plate and incubated for $4 \mathrm{~h}$. Then $2 \times 10^{4}$ KMS12-PE myeloma cells were added to the upper chamber. Incubation was performed for $72 \mathrm{~h}$.

Cultures without KMS12-PE cells served as negative control for transwell cultures and co-cultures.

\section{Detection of SA- $\beta$ GalA}

$\beta$-galactosidase activity was measured as previously reported [36]. Data was acquired as described above and analyzed using the median fluorescence intensity (MFI). Co-cultures of HD-BMMSCs $(\mathrm{n}=3)$ and HS-5 stromal cells $(\mathrm{n}=3$, CRL-11882) were used as controls. In addition $\beta$-galactosidase activity was analyzed using the "Senescence cells Histochemical Staining Kit" (Sigma Aldrich) as recommended by the manufacturer.

\section{Cell cycle analysis}

Cell analysis was performed using the "Cell cycle Assay Kit" (Abcam) as recommended by the manufacturer. Data was acquired using a logarithmic scale.

\section{Quantitative Real-Time PCR (qPCR)}

Total RNA was extracted using TRIzol as described previously [37]. RNA was treated with DNase (Ambion) and poly(A)-polymerase (NEB) according to the manufacturer's instructions. $800 \mathrm{ng}$ of RNA was used for cDNA synthesis with a Transcriptor First Strand cDNA Synthesis Kit (Roche) and $2 \mu \mathrm{l}$ of poly(T)VN adaptor primer $(10 \mathrm{pmol})$ in a $20 \mu \mathrm{l}$ reaction.

qPCR was performed with the FastStart Universal SybrGreen Master Mix (Roche). MicroRNA detection was performed as described [38]. GAP-DH and 5.8S rRNA were chosen as housekeeping genes. All primers and corresponding accession numbers are listed in Additional file 3: Supplemental Methods; Table S2. QPCR was carried out with the RotorGene 6000 Real-Time PCR cycler using 1:5 diluted cDNA (8 ng) as template. Cycling conditions are described in Additional file 2: Supplemental Methods; Table S3. qPCR efficiencies were determined using linear regression analysis $[39,40]$ using LinRegPCR software and relative quantifications were estimated with the Pfaffl-method [41]. Received data was analyzed with the Rotor Gene 6000 software.

\section{Quantitative methylation-specific PCR (qMSP)}

DNA isolation was performed using Puregene reagents (Qiagen) according to the manufacturer's instructions. 300 ng of genomic DNA was subjected to bisulfite treatment with the EpiTect Fast Bisulfite Conversion Kit
(Qiagen) as recommended in the manual. Primers are described in Additional file 2: Supplemental Methods; Table S2. Reactions were performed with $30 \mathrm{ng}$ treated DNA using SYBR Green Master Mix (Roche). Thermal conditions were as described above. Quantification was carried out using a standard curve generated using a dilution series of fully methylated with unmethylated DNA (Applied Biosystems). Each sample was analyzed in duplicates and $\mathrm{Ct}$-values above 32 were excluded.

\section{Copy number (CN) variation assay}

Three genomic regions located along each of the clusters were chosen for $\mathrm{CN}$ estimations of DLK1-DIO3 and C19MC. Assay numbers (qBiomarker Copy Number Assays, Qiagen) are listed in Additional file 2: Supplemental Methods; Table S4. Genomic DNA of the stromal cell line HS-5 (CRL-11882) was applied as a calibrator. Cycling was carried out as recommended by the manufacturer. Relative quantification was achieved by the $\Delta \Delta \mathrm{C}_{\mathrm{t}}$ method as described in the qBiomarker manual.

\section{microRNA mimic and inhibitor experiments}

MM-BMMSCs were seeded in a 6-well plate with 1$2.5^{*} 10^{5}$ cells/well. After incubation for $3 \mathrm{~h}$ cells were transfected with $10 \mathrm{mM}$ of miR-485-5p mimic, $100 \mathrm{mM}$ of miR-485-5p inhibitor or the appropriate negative control. The mimic, inhibitor and corresponding negative controls were all obtained from Qiagen AG. Transfections were performed with the HiPerfect Transfection Reagent (Qiagen) as recommended by the manufacturer. In addition, a transfection control containing only the transfection reagent was carried out. Transfected MM-BMMSCs were incubated for $48 \mathrm{~h}$ before use for downstream analysis.

\section{Indirect enzyme-linked immunosorbent assay (ELISA)}

Complete cell lysates of BMMSCs were prepared using RIPA buffer (Pierce). The protein amount was detected with a BCA Protein Assay Kit (Pierce). $5 \mu \mathrm{g}$ of total protein were used for ELISAs. 96-well plates were coated with the samples overnight at $4^{\circ} \mathrm{C}$ using $\mathrm{BupH}$ coating buffer (Pierce). The coated wells were blocked for $1 \mathrm{~h}$ at room temperature (5\% nonfat dry milk in PBS). Primary antibodies were diluted in blocking buffer and incubation was performed overnight at $4^{\circ} \mathrm{C}$ (cyclin D1 and p21 1:500 (Cell Signaling); cyclin E (sc-481) 1:100 (Santa Cruz); CDKN2A 1:200 (Thermo Scientific)). Between the incubation steps the wells were washed three times with PBS containing $0.1 \%$ Tween. The secondary antibody (anti-rabbit IgG-HRP (Cell Signaling)) was diluted 1:1000 and incubation was performed for $2 \mathrm{~h}$ at room temperature. Detection was performed with TMB substrate (Pierce) and absorption was measured at $450 \mathrm{~nm}$. All measurements were performed with three technical replicates. A dilution series of complete cell lysates of the HS-5 cell line was used 
for standard curve generation enabling relative quantification of protein levels. Pure RIPA buffer served as negative control.

\section{Statistical analysis}

All experiments were statistically analyzed using GraphPad Prism 6 software (LA Jolla, CA, USA). The data shown represents the mean \pm standard error of the mean (SEM). Comparisons of HD-BMMSCs with MM-BMMSCs were performed using the Mann-Whitney U test. The Wilcoxon signed-rank test was used for the analysis of co-cultures and transwell cultures. The unpaired/paired t-test was used for statistical analysis of protein level results (ELISA analysis). Transfection experiments were analyzed using the paired $\mathrm{t}$-test. Results were considered statistically significant when $\mathrm{p} \leq 0.05$.

\section{Results}

BMMSCs from MM patients at the time of diagnosis are referred to as ND-MM-BMMSCs and BMMSCs from relapsed MM patients are referred to as R-MM-BMMSCs. For both analysis groups the abbreviation MM-BMMSCs is used. BMMSCs from healthy donors are referred to as HD-BMMSCs. Because ND-MM-BMMSCs and R-MMBMMSCs showed a similar mRNA expression, no separate analysis of ND-MM-BMMSCs and R-MM-BMMSCs was performed for protein tests.

Given that the observed changes in ND-MM-BMMSCs and R-MM-BMMSCs upon co-cultivation with KMS12PE myeloma cells were similar the data of co-cultures, transwell cultures and microRNA transfection experiments of both groups were combined to one analysis group (MM-BMMSCs).

\section{MM-BMMSCs exhibit a higher senescence state than HD-BMMSCs}

In contrast to HD-BMMSCs, MM-BMMSCs displayed a 2 - to 3 -fold $(p<0.005)$ higher SA- $\beta$ GalA in passage 1 and 4 of the cell cultures (Figure 1A). R-MM-BMMSCs had a 1.4-fold increased SA- $\beta$ GalA as compared to NDMM-BMMSCs. These results could be confirmed by a histological $\beta$-galactosidase staining of HD-BMMSCs and MM-BMMSCs in passage 4 (Figure 1B). Furthermore, qPCR analyses showed a 2.5- to 4-fold lower expression of cyclin E1 and a 5- to 6-fold overexpression of cyclin D1 in ND-MM-BMMSCs and R-MM-BMMSCs compared to HD-BMMSCs $(p<0.05$; Figure $1 C)$. In addition, the cell cycle inhibitor p21 was 2.5-fold upregulated in MMBMMSCs compared to HD-BMMSCs $(p<0.05)$ against what no changes in the mRNA level of p16 were detected. Similar differences between HD-BMMSCs and MMBMMSCs were detected at the protein level (Figure 1D). Cyclin E1 was 2.8-fold decreased in MM-BMMSCs compared to HD-BMMSCs $(p=0.0416)$. In contrast, cyclin D1 and p21 protein levels were 1.5 -fold to 1.8 -fold increased. Protein measurement showed also a slightly reduced level of p16 in MM-BMMSCs but this change was below 1.5fold. These results correlated with a higher amount of cells in $S$ phase and a reduced amount of cells in $G_{1} / G_{0}$ phase compared to HD-BMMSCs $(p<0.008$; Figure 1E).

\section{MicroRNAs in MM-BMMSCs are aberrantly expressed}

To investigate whether senescence of MM-BMMSCs is correlated with changes in the microRNA expression, the level of different microRNAs was analyzed using qPCR. We chose six microRNAs, which were previously reported to be deregulated in MM cells and to play a possible role in the generation of senescence or cell cycle arrest (miR16, miR-485-5p, miR-519d, miR-221, miR-126, miR-223). Analysis revealed an overexpression of miR-16, miR-223, miR-485-5p and miR-519d (all with $p<0.025$ ) in MMBMMSCs compared to HD-BMMSCs. No expression differences were detected for miR-221 and miR-126 (Figure 2A).

\section{MM-BMMSCs show hypomethylation and copy number accumulation of the DLK1-DIO3 and C19MC genomic clusters}

qPCR analysis revealed the overexpression of miR-485$5 \mathrm{p}$ and miR-519d in MM-BMMSCs. These microRNAs are located on two imprinted clusters on chromosomes 14 (DLK1-DIO3) and 19 (C19MC), respectively, and are reported to play a role in senescence generation $[21,31,32]$. Given that expression of both clusters is controlled by methylation of their regulatory regions, we analyzed their methylation status using qMSP (Figure 2B).

Hypomethylation of both clusters in MM-BMMSCs compared to HD-BMMSCs was observed (Figure 2C). For DLK1-DIO3, MM-BMMSCs exhibited an approximate 5-fold lower methylation level of the IG-DMR. ND-BMMSCs methylation levels were not statistically different for C19MC ( $p=0.0537)$. However, they exhibited the same characteristics as for R-MM-BMMSCs $(p=0.0062)$ with a 2.5 -fold lower methylation level compared to HD-BMMSCs.

Another reason for the overexpression of miR-485-5p and miR-519d could be $\mathrm{CN}$ variations of C19MC and DLK1-DIO3. CN variation analysis was carried out at three regions along each cluster (Figure 2B).

MM-BMMSCs displayed high levels of amplification of the backward region of C19MC (Figure 2D). Error bars indicate the detection of individual MM-BMMSCs with a $\mathrm{CN}>7$. The front and middle regions of $\mathrm{C} 19 \mathrm{MC}$ exhibited 3 to 4 copies in MM-BMMSCs whereas in HD-BMMSCs detected CNs were below $2.7(p<0.05)$. DLK1-DIO3 was also commonly amplified, especially the front and the back regions (Figure 2E). Copy number alterations for R-MMBMMSCs in the middle region of DLK1-DIO3 were not 


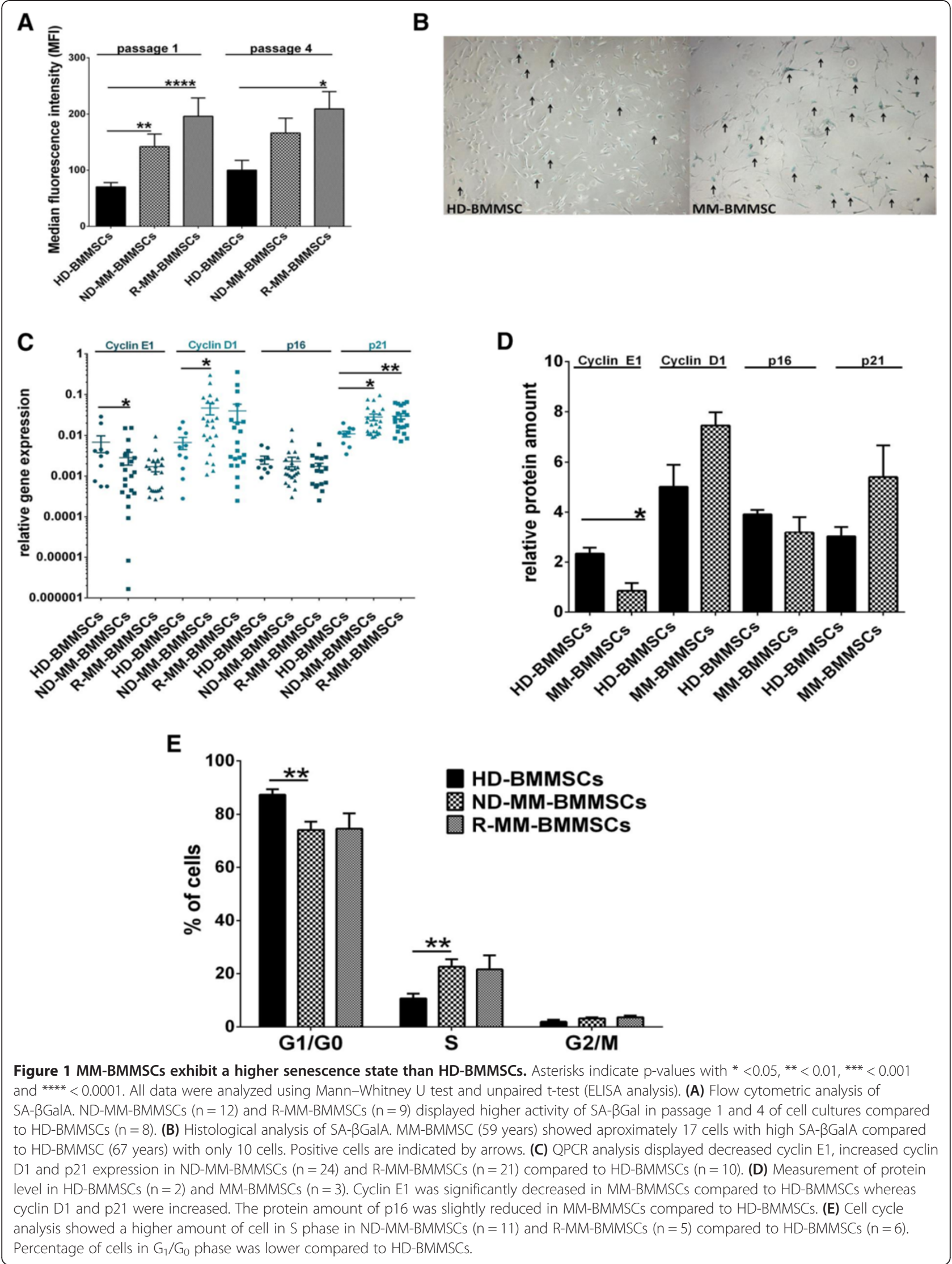




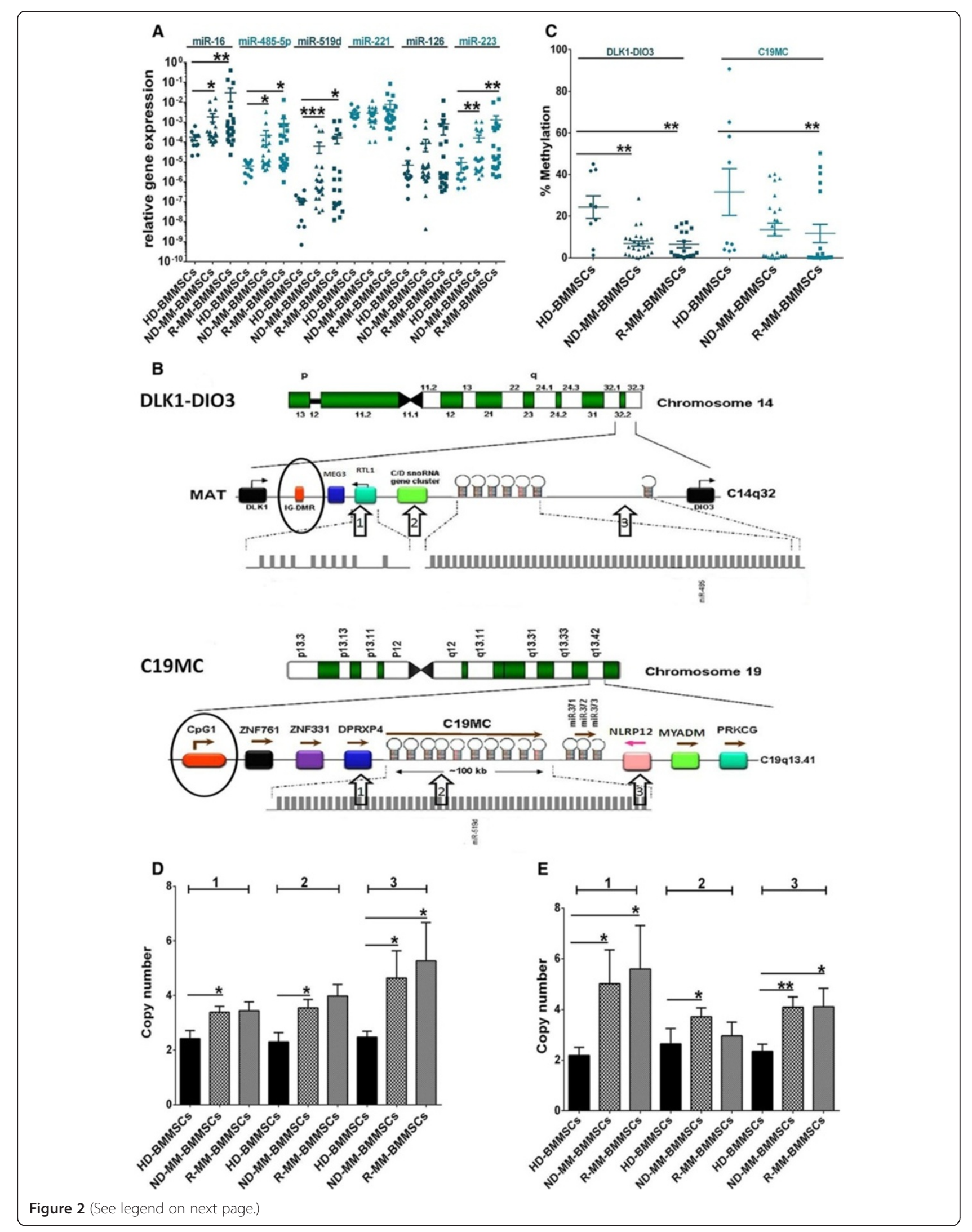


(See figure on previous page.)

Figure 2 Overexpressed microRNAs in MM-BMMSCs are associated with hypomethylation and CN accumulation of DLK1-DIO3 and C19MC. Asterisks indicate $p$-values with ${ }^{*}<0.05,{ }^{* *}<0.01,{ }^{* * *}<0.001$ and ${ }^{* * *}<0.0001$. All data were analyzed using Mann-Whitney $U$ test. (A) ND-MM-BMMSCs $(n=24)$ and R-MM-BMMSCs $(n=21)$ showed high overexpression of miR-16, miR-485-5p, miR-519d and miR-223 compared to HD-BMMSCs $(n=10)$. (B) Schematic presentation of the genomic organization of DLK1-DIO3 on chromosome 14 (C14q32) and C19MC on chromosome 19 (C19q13.41). Circles indicate the regulatory region of the respective cluster and arrows point to positions of CN measurement. Modified from Morales-Prieto et al. [27] (C) The regulatory regions of DLK1-DIO3 and C19MC were hypomethylated in ND-MM-BMMSCs ( $n=25)$ and R-MM-BMMSCs $(n=18)$ compared to HD-BMMSCs $(n=9)$. (D) CN analysis of C19MC displayed CN accumulation in all three regions in ND-MM-BMMSCs $(n=23)$ and R-MM-BMMSCs $(n=15)$ compared to HD-BMMSCs $(n=8)$. $(E) C N$ analysis of DLK1-DIO3 displayed CN accumulation in all three measured positions in MM-BMMSCs compared to HD-BMMSCs (sample number as indicated in (D)). No accumulation was measured for R-MM-BMMSCs in position 2.

detectable $(p=0.6466)$, though ND-MM-BMMSCs exhibited significant amplification $(p=0.0065)$.

\section{KMS12-PE myeloma cells reduce SA- $\beta$ GalA and modify cell cycle characteristics of MM-BMMSCs}

Co-cultures and transwell cultures of the KMS12-PE cell line with MM-BMMSCs were carried out to analyze whether MM cells can exert an influence on the senescence characteristics of MM-BMMSCs (Figure 3A). When co-cultured with myeloma cells MM-BMMSCs exhibited a reduced SA- $\beta$ GalA compared to the same MM-BMMSCs cultured alone (median fluorescence intensity (MFI) of 118.1 vs. $178.4 ; p<0.0001)$. A similar effect could be detected using transwell cultures to prevent cell-cellcontact between MM-BMMSCs and KMS12-PE cells (MFI of 173 vs. 267.3; $p<0.0313$ ). No effect on the activity of SA- $\beta$ Gal was observed in HD-BMMSCs and the HS-5 cell line co-cultured with KMS12-PE myeloma cells. In addition, mRNA expression of co-cultured and transwell cultured MM-BMMSCs was measured (Figure 3B). No effect could be detected for cyclin D1 and p16, whereas cyclin E1 was 3.25-fold upregulated in co-cultured and 1.6-fold upregulated in transwell cultured MM-BMMSCs $(p<0.05)$. Surprisingly, cell interaction with MM cells also induced an upregulation of p21 mRNA in MM-BMMSCs. This effect was lower in transwell cultured MM-BMMSCs compared to co-cultured MM-BMMSCs (1.9-fold and 3fold; $p<0.008)$. However, contrary results were detected at protein level showing a 1.7-fold reduction of p21 in cocultured MM-BMMSCs (Figure 3C). In addition, cyclin D1 protein expression was 1.8 -fold reduced upon cocultivation with KMS12-PE myeloma cells whereas no change was seen on mRNA level $(p=0.0033)$. In contrast, the mRNA and protein analysis of cyclin E1 and p16 was concordant. Co-cultured MM-BMMSCs displayed also 2fold upregulation of cyclin E1 on protein level against what the protein level of p16 was unchanged. In this context cell cycle distribution of co-cultured and transwell cultured MM-BMMSCs was analyzed (Figure 3D). Both cell culture systems led to a slight reduction of cells in S phase compared to MM-BMMSCs cultured alone $(p=0.0078)$. Furthermore, a light increase of cells in $G_{1} / G_{0}$ phase was detected for co-cultured MMBMMSCs compared to MM-BMMSCs cultured alone $(p=0.0078)$. Transwell cultured MM-BMMSCs showed the same tendency but significant changes were not detectable.

\section{Cell interaction between KMS12-PE myeloma cells and MM-BMMSCs induces changes of microRNA expression in both cell types}

To explore whether changes in senescence and cell cycle characteristics of MM-BMMSCs upon cultivation with KMS12-PE cells are associated with changes in the microRNA level of overexpressed microRNAs (miR-16, miR-223, miR-485-5p and miR-519d), the microRNA expression of co-cultured and transwell cultured MM-BMMSCs was measured using qPCR (Figure 4A). Significant changes were detected for miR-223 and miR-485-5p. MiR-223 was 3-fold downregulated in co-cultured MM-BMMSCs $(p<0.007)$, whereas no effect was detected in transwell cultured MM-BMMSCs. In contrast, downregulation of miR-485-5p was detected in both cell culture systems, whereby the effect reduced from 4-fold in co-cultured MM-BMMSCs to 2-fold in transwell cultured MMBMMSCs $(p<0.03)$. Interestingly, cell-cell-interaction also altered microRNA expression of KMS12-PE myeloma cells. MiR-221 increased 13.78-fold whereas miR-223 and miR-519d were 2.72 - to 2.85 -fold reduced in co-cultured KMS12-PE myeloma cells ( $<<0.02$, Figure 4B). Expression of miR-485-5p was not detectable in KMS12-PE myeloma cells.

Furthermore, we analyzed whether changes in the methylation status of the DLK1-DIO3 regulatory region upon co-cultivation with KMS12-PE cells could be the reason for changes in miR-485-5p expression (Figure 4C). In co-cultured MM-BMMSCs a significant increase in the percentage IG-DMR methylation compared to MMBMMSCs cultured alone was detected $(14.31 \pm 3.741 \%$ vs. $9.931 \pm 2.21 \% ; p<0.004)$. In contrast, no $\mathrm{CN}$ variations of DLK1-DIO3 in co-cultured MM-BMMSCs compared to MM-BMMSCs cultured alone were detected (data not shown). 


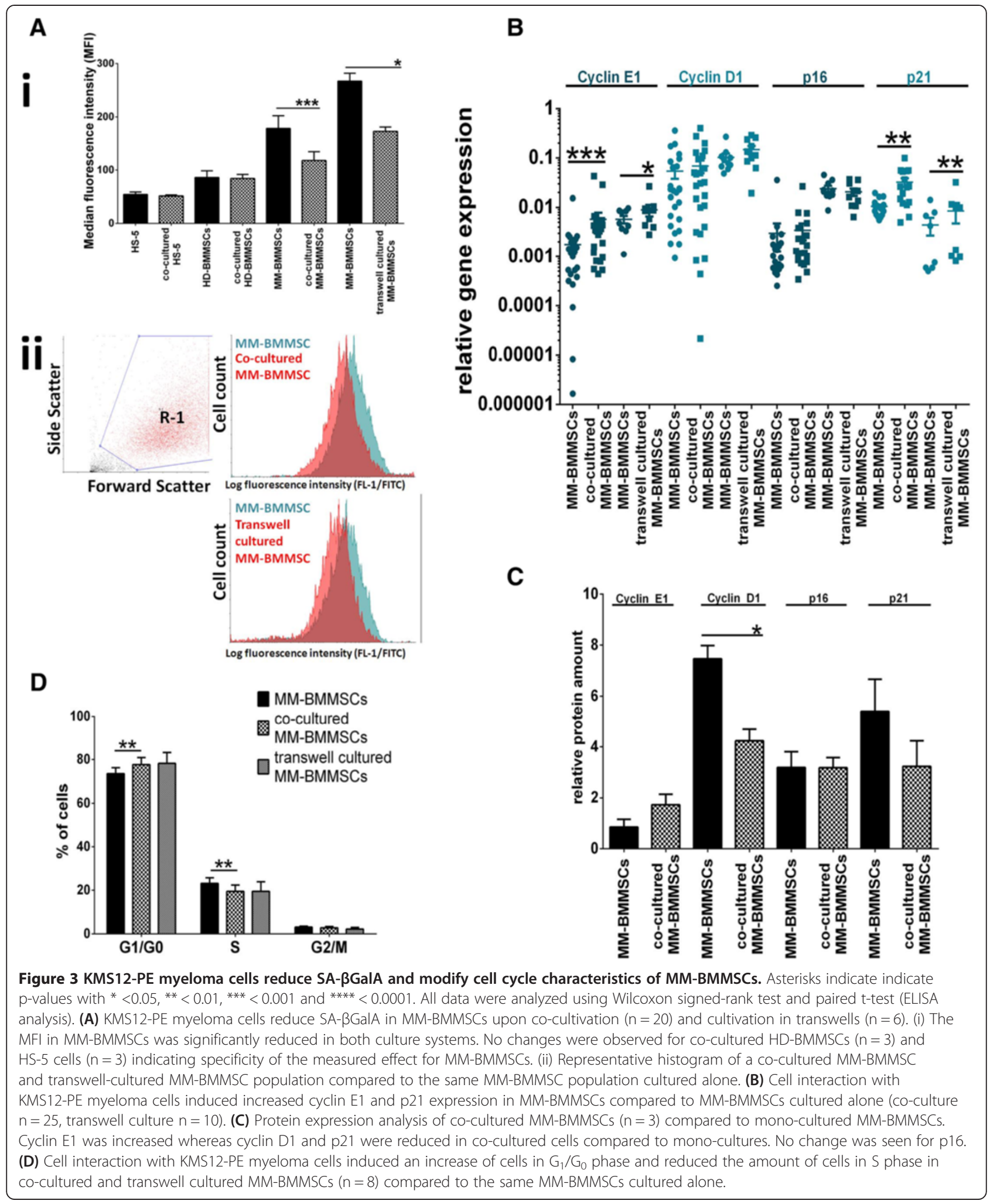

MiR-485-5p modifies senescence and cell cycle characteristics of MM-BMMSCs

To explore whether downregulation of miR-485-5p is associated with the reduction of cells in S phase and SA- $\beta$ GalA of MM-BMMSCs, transfections with miR-485-5p mimic or inhibitor were performed. Transient overexpression of miR-485-5p induced a reduction of SA- $\beta$ GalA, whereas the inhibition of miR-485-5p led to an increase in 

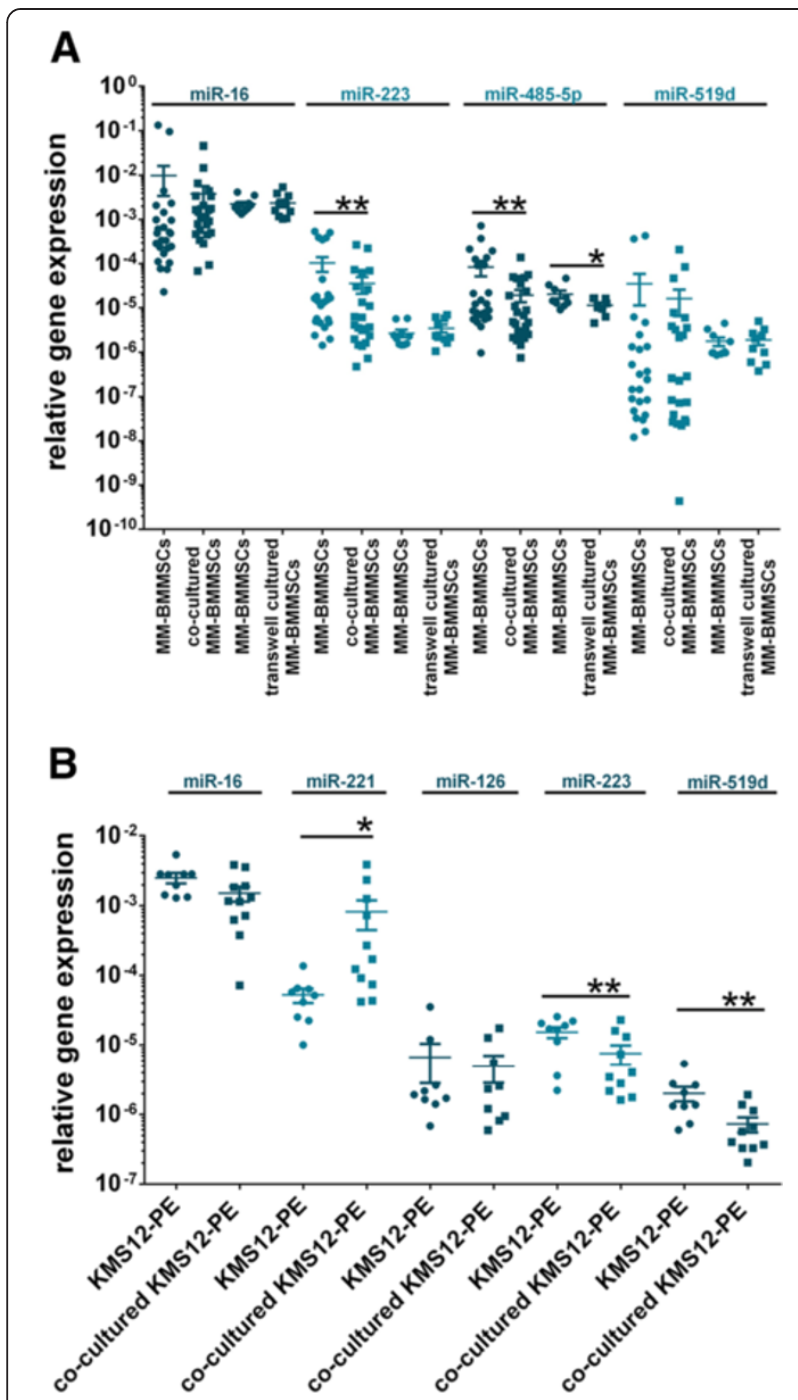

C

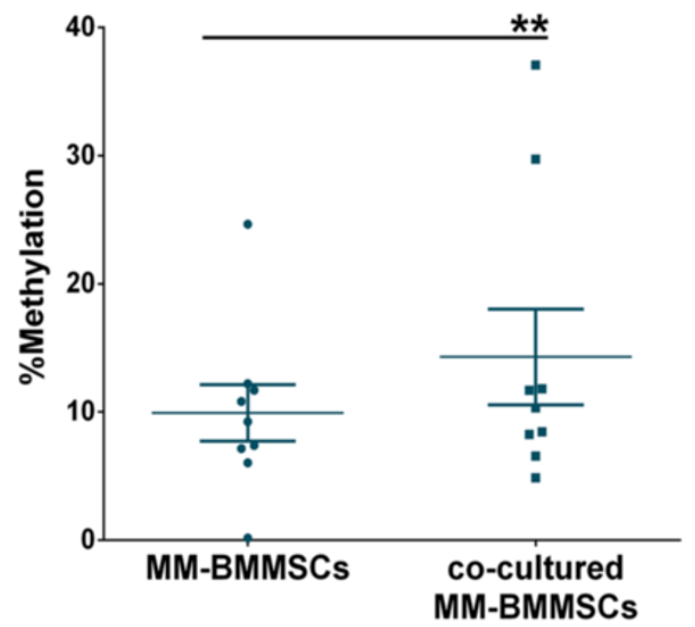

Figure 4 KMS12-PE myeloma cells downregulate miR-223 and miR-485-5p in MM-BMMSCs. Asterisks indicate indicate $p$-values with ${ }^{*}<0.05,{ }^{* *}<0.01,{ }^{* * *}<0.001$ and ${ }^{* * *}<0.0001$. All data were analyzed using Wilcoxon signed-rank test. (A) Co-cultured MM-BMMSCs $(n=25)$ displayed reduced expression of miR-223 and miR-485-5p. Transwell-cultured $(n=10)$ MM-BMMSCs showed no changes in miR-223 expression but also decreased miR-485-5p levels. Intensity of changes in miR-485-5p decreased when cell-cell-contact was prevented by transwell cultivation. (B) Cell interaction with MM-BMMSCs induced changes in the microRNA expression of KMS12-PE myeloma cells $(n=10)$. MiR-221 was upregulated whereas miR-223 and miR-519d decreased in co-cultured KMS12-PE myeloma cells. (C) Cell interaction with KMS12-PE myeloma cells led to increased methylation of the IG-DMR of DLK1-DIO3 as measured by qMSP $(n=9)$.

the MFI compared to the negative and transfection controls $(p<0.05$; Figure $5 \mathrm{~A})$. Overexpression of miR-485-5p had no effect on the cell cycle of MM-BMMSCs (data not shown), whereas inhibition of miR-485-5p induced different effects in MM-BMMSCs. Three MM-BMMSCs out of five transfected with the miR-485-5p inhibitor showed a decrease of cells in $S$ phase and a significant increase of cells in $\mathrm{G}_{1} / \mathrm{G}_{0}$ phase compared to the controls $(p<0.003$; Figure 5B). In contrast, two MM-BMMSCs displayed an inverse effect with an increase of cells in $\mathrm{S}$ phase and a decrease of cells in $G_{1} / G_{0}$ phase ( $p<0.008$; Figure $5 C$ ). These diverse results displayed no correlation with the ND-MM-BMMSCs or R-MM-BMMSCs characteristic.

\section{Discussion}

MM-BMMSCs play a critical role in MM tumor growth and survival. Several studies suggest the existence of senescence-associated constitutive abnormalities in MMBMMSCs, and that these lead to abnormal cell characteristics and increased tumor support [11-13,15,42,43]. In this study we investigated the correlation between senescence and microRNA expression in MM-BMMSCs and the influence of MM cells on these alterations.

In vitro cultured MM-BMMSCs showed increased senescence and accumulation of cells in $\mathrm{S}$ phase. These characteristics were associated with a reduced cyclin E1 and an increased cyclin D1 expression. R-MM-BMMSCs showed a higher SA- $\beta$ GalA compared to ND-MM-BMMSCs. Therefore, it seems that relapse of MM could increase the premature senescence phenotype of MM-BMMSCs. In addition, the increased activity of SA- $\beta$ GalA could be associated with the prolonged disease progression which is present in relapsed patients. As previously described we found overexpression of $\mathrm{p} 21$ but not $\mathrm{p} 16$ pointing to DNA damage mediated senescence [15]. Though senescence of MM-BMMSCs was already reported the reasons for this cellular state are not clear $[12,14,15,43]$.

MicroRNAs are potential regulators of senescence but data relating to microRNA expression in MM-BMMSCs 


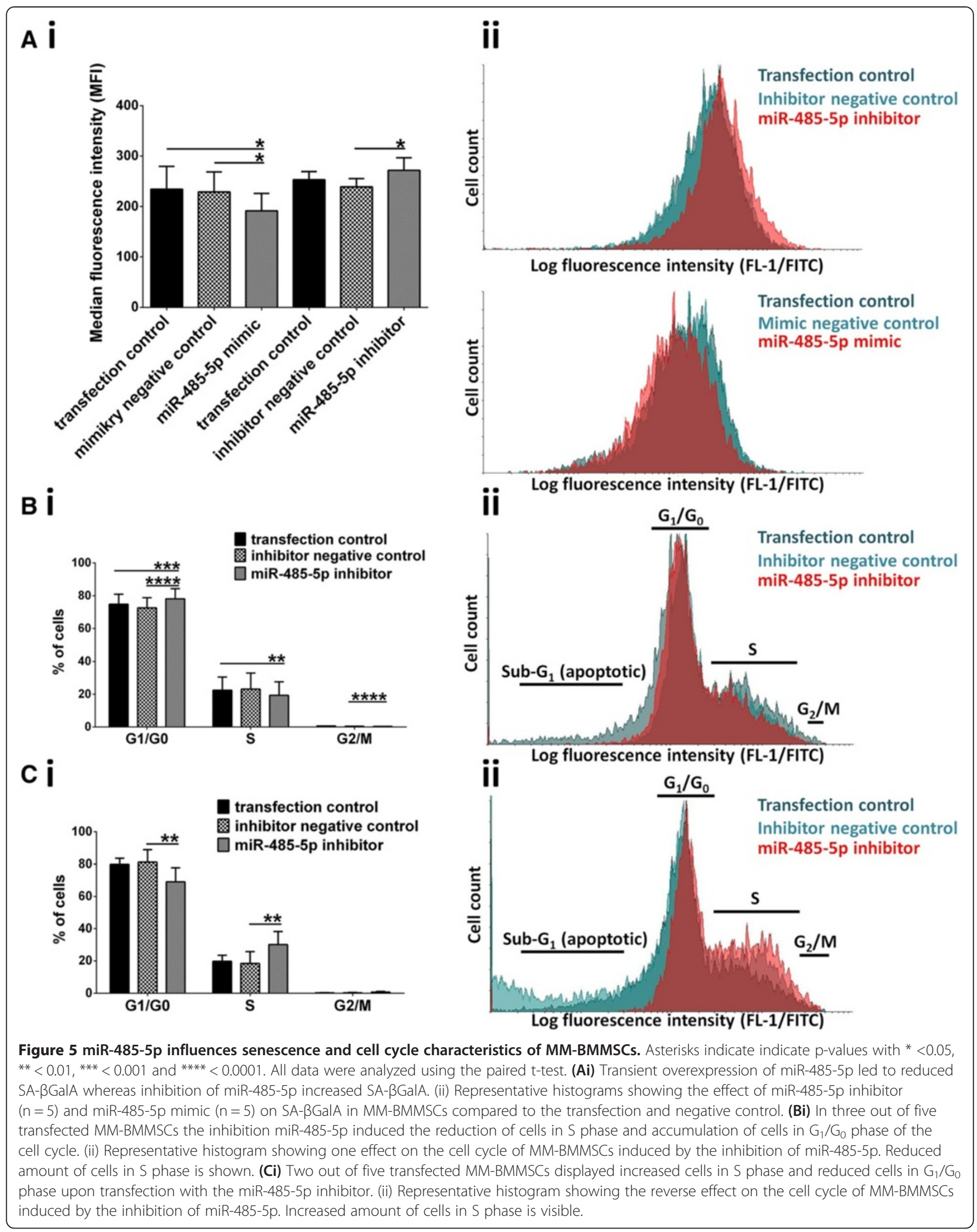


is limited [44]. Here, six microRNAs chosen on the basis of the microarray data from MM cells were analyzed [29]. Overexpression was found for miR-223, which was also overexpressed in MM cells. Furthermore miR-16, miR-485-5p and miR-519d were overexpressed in MMBMMSCs whereas these microRNAs were downregulated in MM cells. Previous studies have identified miR-16 as a tumor suppressor in leukemic cells and its overexpression in MM-BMMSCs could induce a similar effect [45]. In contrast, no specific functions of miR-223, miR-485-5p and miR-519d in MM are known. MiR-485-5p and miR$519 \mathrm{~d}$, which are expressed on the clusters DLK1-DIO3 and $\mathrm{C} 19 \mathrm{MC}$, were reported to play a role in senescence generation $[21,32,46,47]$. The regulatory regions of both clusters were hypomethylated together with $\mathrm{CN}$ accumulation of the genomic regions in MM-BMMSCs. These two alterations could be responsible for the overexpression of miR-485-5p and miR-519d. The aberration of genomic $\mathrm{CN}$ also points to genomic alterations in $\mathrm{MM}$ BMMSCs possibly explaining the p21-associated senescence state. In addition, Abdeolmohsen et al. reported that miR-519 promotes DNA damage and elevates the level of p21 [32].

Next, we analyzed whether cell interaction with MM cells influences these alterations. Surprisingly, cell interaction with KMS12-PE cells led to reduced SA- $\beta$ GalA and cells in $\mathrm{S}$ phase of the cell cycle. This effect was also seen when cell-cell-contact was prevented by transwells. Thus, the increase of cells in $G_{1} / G_{0}$ phase points to a proproliferative influence of MM cells on MM-BMMSCs. This shift in the cell cycle characteristics could also be the reason for the decreased cyclin D1 expression and the increased expression of cyclin E1 in co-cultured MMBMMSCs. Noll et al. reported that MM cells alter the microenvironment of MM by supporting the proliferation of mesenchymal stem cells through soluble factors as IL-6 [48]. Therefore oncogenic signaling by MM cells by different cytokines could induce the reversal of senescence in MM-BMMSCs [49]. In addition, the reduction of SA$\beta$ GalA was accompanied by a reduction of $\mathrm{p} 21$ at the protein level. In contrast, p21 mRNA expression was increased. The mRNA amount is not a direct reflection of protein expression since post-transcriptional processes are significant to the final synthesis of proteins. Contrary expression levels of mRNA and proteins could be of interest for the understanding of the interaction between myeloma cells and BMMSCs in MM pathogenesis. Here, it could be induced by a feedback mechanism activated through the depletion of $\mathrm{p} 21$ at the protein level. In addition, the reversal mechanisms of senescence are still not clear $[17,18]$. Even though senescence in MM-BMMSCs seems to be generated by increased p21 expression it is possible that reversal of this cellular state is mediated by a distinct signaling pathway $[31,45]$.
Cell interaction with MM cells reduced levels of miR223 and miR-485-5p in MM-BMMSCs. Given that only miR-485-5p was also reduced when cell-cell-contact between MM-BMMSCs and MM cells was prevented, this microRNA was a potential candidate for modulation of senescence in MM-BMMSCs. In addition, cell interaction seems to increase methylation of the IG-DMR of DLK1-DIO3 possibly leading to reduced expression of the cluster-associated microRNAs [50]. Interaction of KMS12PE myeloma cells with MM-BMMSCs also induced microRNA changes in the MM cell fraction. Cell-cell-interaction strongly increased the level of miR-221 in KMS12-PE myeloma cells. This microRNA acts as an oncogene by downregulation of different cell cycle inhibitors leading to an increased tumor cell proliferation [51]. In addition, miR-223 and miR-519d were downregulated in co-cultured KMS12-PE myeloma cells. As previously mentioned these microRNAs could have anti-proliferative effects on cells $[21,32,46,47]$. Therefore, interaction with MM-BMMSCs seems to shift microRNA levels of KMS12-PE myeloma cells towards a more pro-proliferative gene expression pattern.

Finally, MM-BMMSCs were transfected with miR-485$5 p$ mimic or inhibitor. The effect on SA- $\beta$ GalA was contrary to the one seen by cell interaction with MM cells because increased miR-485-5p levels decreased the SA$\beta$ GalA and vice versa. This indicates that miR-485-5p alone is not responsible for reversal of senescence in MM-BMMSCs but additional genetic events are needed. This could be based on the fact that increased methylation of the IG-DMR of DLK1-DIO3, which possibly leads to reduced transcription of this genomic region, controls all associated microRNAs. Therefore, the mechanism which induces the observed effect in MM-BMMSCs upon cell interaction with MM cells could be rather complex. Interestingly, inhibition of miR-485-5p also induced different cell cycle effects in MM-BMMSCs. Three of the transfected MM-BMMSCs displayed an effect which was similar to the one induced by cell interaction with MM cells, whereas the other two showed a contrary cell cycle change. This again indicates that miR-485-5p acts in dependence of additional molecular genetic events or cellular properties [47].

Although manipulation of miR-485-5p alone could not mimic the effect on SA- $\beta$ GalA and the cell cycle of MMBMMSCs upon cell interaction with MM cells, the results strongly indicate that miR-485-5p and the associated cluster DLK1-DIO3 participate in the regulation of senescence and cell cycle characteristics in MM-BMMSCs. Therefore, further investigations of this topic are needed. Furthermore, the premature senescence phenotype of MMBMMSCs seems to be an attendant phenomenon rather than a specific tumor supportive stromal cell characteristic. The reduction of the senescence phenotype by 
MM cells indicates that interaction of MM cells and MM-BMMSCs does not rely on this cell characteristic. It is likely that MM cells benefit from a more vital cellular state of MM-BMMSCs. Therefore, the premature senescence phenotype of MM-BMMSCs could be a side effect possibly induced through increased activation of stromal cells by MM cells. However, further analysis is needed to determine whether the senescence-associated constitutive changes of MM-BMMSCs could support relapse of MM disease.

\section{Conclusions}

In summary we found that senescence in MM-BMMSCs is associated with the overexpression of potential senescencemediating microRNAs and copy number variations of DLK1-DIO3 and C19MC. This is the first report indicating that the senescence phenotype of MM-BMMSCs is partially reversed by cell interaction with MM cells and it is therefore not clear whether this phenotype could in vivo be important for the pathogenesis of active MM disease or is more important when cell interaction of MM-BMMSCs with MM cells is inhibited. More investigations regarding the role of senescence of MM-BMMSCs in MM could lead to new insights for the improvement of therapy efficiency and follow-up treatment of MM patients. The genomic region of DLK1-DIO3 includes potential senescence-modulating microRNAs and further analysis of the role of DLK1-DIO3 and the associated microRNAs could reveal new insights into the genetic events that senescence relies on.

\section{Additional files}

Additional file 1: Table S1. Patients and Donor characteristics.

Additional file 2: Figure S1. Purity of isolated BMMSCs. BMMSCs were double stained with CD34-PE/CD90-FITC or CD45-PE/CD105-FITC. Purity of BMMSCs ranged from $94 \%$ to $99.5 \%$ in passage 1 of cell cultures.

Additional file 3: Table S2. Primers used in this study. Table S3. Cycling conditions for qPCR. Table S4. qBiomarker Assays used for CN variation analysis.

\footnotetext{
Abbreviations

MM: Multiple myeloma; MM-BMMSCs: Multiple myeloma bone marrow stromal cells; ND-MM-BMMSCs: Newly diagnosed MM-BMMSCs; R-MMBMMSCs: Relapsed MM-BMMSCs; SA- $\beta$ GalA: Senescence-associated $\beta$-galactosidase activity.
}

\section{Competing interests}

The authors declare that they have no competing interests.

\section{Authors' contribution}

BR participated in the design of the study and the cell cultivation, performed the molecular genetic analysis and drafted the manuscript. BO and BIW conceived of the study, participated in its design and coordination and helped to draft the manuscript. NA, SHM, KA and BIW participated in the collection of patient samples. WM participated in the cell cultivation. SE participated in the molecular genetic analysis. PA and DB conceived of the study and participated in its coordination. All authors read and approved the final manuscript.

\section{Acknowledgments}

This work was supported by the Stefan-Morsch-Stiftung for Leukemia Tumor Patients.

\section{Author details}

${ }^{1}$ Department of Hematology, Oncology and Tumourimmunology, Charité Universitätsmedizin Berlin, Hindenburgdamm 30, 12200 Berlin, Germany. ${ }^{2}$ Department of Hematology, Oncology and Tumourimmunology, Helios Clinic Berlin-Buch, Berlin, Germany.

Received: 24 October 2014 Accepted: 10 February 2015

Published online: 18 February 2015

\section{References}

1. Chesi M, Bergsagel PL. Molecular pathogenesis of multiple myeloma: basic and clinical updates. Int J Hematol. 2013;97(3):313-23.

2. Hideshima T, Mitsiades C, Tonon G, Richardson PG, Anderson KC. Understanding multiple myeloma pathogenesis in the bone marrow to identify new therapeutic targets. Nat Rev Cancer. 2007;7(8):585-98.

3. Katz BZ. Adhesion molecules-the lifelines of multiple myeloma cells. Semin Cancer Biol. 2010;20(3):186-95.

4. Manier S, Sacco A, Leleu X, Ghobrial IM, Roccaro AM. Bone marrow microenvironment in multiple myeloma progression. J Biomed Biotechnol. 2012;2012:157496.

5. Gilmore TD. Multiple myeloma: lusting for NF-kappaB. Cancer Cell. 2007;12(2):95-7.

6. Li ZW, Chen H, Campbell RA, Bonavida B, Berenson JR. NF-kappaB in the pathogenesis and treatment of multiple myeloma. Curr Opin Hematol. 2008;15(4):391-9.

7. Hideshima T, Bergsagel PL, Kuehl WM, Anderson KC. Advances in biology of multiple myeloma: clinical applications. Blood. 2004;104(3):607-18.

8. Landowski TH, Olashaw NE, Agrawal D, Dalton WS. Cell adhesion-mediated drug resistance (CAM-DR) is associated with activation of NF-kappa B (RelB/p50) in myeloma cells. Oncogene. 2003;22(16):2417-21.

9. Neri P, Ren L, Azab AK, Brentnall M, Gratton K, Klimowicz AC, et al. Integrin beta7-mediated regulation of multiple myeloma cell adhesion, migration, and invasion. Blood. 2011;117(23):6202-13.

10. Shain KH, Yarde DN, Meads MB, Huang M, Jove R, Hazlehurst LA, et al. Beta1 integrin adhesion enhances IL-6-mediated STAT3 signaling in myeloma cells: implications for microenvironment influence on tumor survival and proliferation. Cancer Res. 2009;69(3):1009-15.

11. Garayoa M, Garcia JL, Santamaria C, Garcia-Gomez A, Blanco JF, Pandiella A, et al. Mesenchymal stem cells from multiple myeloma patients display distinct genomic profile as compared with those from normal donors. Leukemia. 2009;23(8):1515-27.

12. Garderet L, Mazurier C, Chapel A, Ernou I, Boutin L, Holy X, et al. Mesenchymal stem cell abnormalities in patients with multiple myeloma. Leuk Lymphoma. 2007:48(10):2032-41.

13. Reagan MR, Ghobrial IM. Multiple myeloma mesenchymal stem cells: characterization, origin, and tumor-promoting effects. Clin Cancer Res. 2012;18(2):342-9.

14. Wallace $\mathrm{SR}$, Oken MM, Lunetta KL, Panoskaltsis-Mortari A, Masellis AM. Abnormalities of bone marrow mesenchymal cells in multiple myeloma patients. Cancer. 2001;91(7):1219-30.

15. Andre T, Meuleman N, Stamatopoulos B, De Bruyn C, Pieters K, Bron D, et al. Evidences of early senescence in multiple myeloma bone marrow mesenchymal stromal cells. PLoS One. 2013;8(3):e59756.

16. Coppe JP, Desprez PY, Krtolica A, Campisi J. The senescence-associated secretory phenotype: the dark side of tumor suppression. Annu Rev Pathol. 2010;5:99-118

17. Campisi J, D'Adda-di-Fagagna F. Cellular senescence: when bad things happen to good cells. Nat Rev Mol Cell Biol. 2007;8(9):729-40.

18. van Deursen JM. The role of senescent cells in ageing. Nature 2014;509(7501):439-46.

19. Fei C, Zhao Y, Guo J, Gu S, Li X, Chang C. Senescence of bone marrow mesenchymal stromal cells is accompanied by activation of p53/p21 pathway in myelodysplastic syndromes. Eur J Haematol. 2014;96(6):476-86.

20. Chen J, Wang M, Guo M, Xie Y, Cong YS. miR-127 regulates cell proliferation and senescence by targeting BCL6. PLoS One. 2013;8(11):e80266. 
21. Marasa BS, Srikantan S, Martindale JL, Kim MM, Lee EK, Gorospe M, et al. MicroRNA profiling in human diploid fibroblasts uncovers miR-519 role in replicative senescence. Aging (Albany NY). 2010;2(6):333-43.

22. Nidadavolu LS, Niedernhofer $L J$, Khan SA. Identification of microRNAs dysregulated in cellular senescence driven by endogenous genotoxic stress. Aging (Albany NY). 2013;5(6):460-73

23. Stadtfeld M, Apostolou E, Ferrari F, Choi J, Walsh RM, Chen T, et al. Ascorbic acid prevents loss of DIk1-Dio3 imprinting and facilitates generation of all-iPS cell mice from terminally differentiated B cells. Nat Genet. 2012:44(4):398-405. S391-392.

24. da Rocha ST, Edwards CA, Ito M, Ogata T, Ferguson-Smith AC. Genomic imprinting at the mammalian Dlk1-Dio3 domain. Trends Genet. 2008:24(6):306-16.

25. Edwards CA, Mungall AJ, Matthews L, Ryder E, Gray DJ, Pask AJ, et al. The evolution of the DLK1-DIO3 imprinted domain in mammals. PLoS Biol. 2008;6(6):e135.

26. Flor I, Bullerdiek J. The dark side of a success story: microRNAs of the C19MC cluster in human tumours. J Pathol. 2012;227(3):270-4

27. Morales-Prieto DM, Ospina-Prieto S, Chaiwangyen W, Schoenleben M, Markert UR. Pregnancy-associated miRNA-clusters. J Reprod Immunol. 2013;97(1):51-61

28. Berenstein R, Blau IW, Nogai A, Wächter M, Pezzutto A, Dörken B, et al. Aberrant expression Of miRNA and mRNA Of cell cycle and adhesion-related genes in bone marrow stroma cells derived from patients with multiple myeloma. Blood. 2013;122(21):3149.

29. Chi J, Ballabio E, Chen XH, Kusec R, Taylor S, Hay D, et al. MicroRNA expression in multiple myeloma is associated with genetic subtype, isotype and survival. Biol Direct. 2011;6:23.

30. Pichiorri F, Suh SS, Ladetto M, Kuehl M, Palumbo T, Drandi D, et al. MicroRNAs regulate critical genes associated with multiple myeloma pathogenesis. Proc Natl Acad Sci U S A. 2008;105(35):12885-90.

31. Olivieri F, Rippo MR, Monsurro V, Salvioli S, Capri M, Procopio AD, et al. MicroRNAs linking inflamm-aging, cellular senescence and cancer. Ageing Res Rev. 2013;12(4):1056-68.

32. Abdelmohsen K, Srikantan S, Tominaga K, Kang MJ, Yaniv Y, Martindale JL, et al. Growth inhibition by miR-519 via multiple p21-inducing pathways. Mol Cell Biol. 2012;32(13):2530-48

33. Najar M, Rouas R, Raicevic G, Boufker HI, Lewalle P, Meuleman N, et al Mesenchymal stromal cells promote or suppress the proliferation of $T$ lymphocytes from cord blood and peripheral blood: the importance of low cell ratio and role of interleukin-6. Cytotherapy. 2009;11(5):570-83.

34. Blau O. Bone marrow stromal cells in the pathogenesis of acute myeloid leukemia. Front Biosci (Landmark Ed). 2014;19:171-80.

35. Blau O, Baldus CD, Hofmann WK, Thiel G, Nolte F, Burmeister T, et al Mesenchymal stromal cells of myelodysplastic syndrome and acute myeloid leukemia patients have distinct genetic abnormalities compared with leukemic blasts. Blood. 2011;118(20):5583-92.

36. Noppe G, Dekker P, de Koning-Treurniet C, Blom J, van Heemst D, Dirks RW, et al. Rapid flow cytometric method for measuring senescence associated beta-galactosidase activity in human fibroblasts. Cytometry A. 2009;75(11):910-6.

37. Rio DC, Ares Jr M, Hannon GJ, Nilsen TW. Purification of RNA Using TRIzol (TRI Reagent). NY, USA: CSHL Press, Cold Spring Harbor; 2010.

38. Shi $R$, Chiang VL. Facile means for quantifying microRNA expression by real-time PCR. Biotechniques. 2005;39(4):519-25.

39. Ramakers $\mathrm{C}$, Ruijter JM, Deprez RH, Moorman AF. Assumption-free analysis of quantitative real-time polymerase chain reaction (PCR) data. Neurosci Lett. 2003;339(1):62-6.

40. Ruijter JM, Ramakers C, Hoogaars WM, Karlen Y, Bakker O, van den Hoff MJ, et al. Amplification efficiency: linking baseline and bias in the analysis of quantitative PCR data. Nucleic Acids Res. 2009;37(6):e45.

41. Pfaffl MW. A new mathematical model for relative quantification in real-time RT-PCR. Nucleic Acids Res. 2001:29(9):e45.

42. Frassanito MA, Rao L, Moschetta M, Ria R, Di Marzo L, De Luisi A, et al. Bone marrow fibroblasts parallel multiple myeloma progression in patients and mice: in vitro and in vivo studies. Leukemia. 2013;28(4):904-16.

43. Corre J, Mahtouk K, Attal M, Gadelorge M, Huynh A, Fleury-Cappellesso S, et al. Bone marrow mesenchymal stem cells are abnormal in multiple myeloma. Leukemia. 2007;21(5):1079-88.

44. Feliciano A, Sanchez-Sendra B, Kondoh H, Lleonart ME. MicroRNAs regulate key effector pathways of senescence. J Aging Res. 2011:2011:205378.
45. Gao SM, Xing CY, Chen CQ, Lin SS, Dong PH, Yu FJ. miR-15a and miR-16-1 inhibit the proliferation of leukemic cells by down-regulating WT1 protein level. J Exp Clin Cancer Res. 2011;30:110

46. Dolezalova D, Mraz M, Barta T, Plevova K, Vinarsky V, Holubcova Z, et al. MicroRNAs regulate p21(Waf1/Cip1) protein expression and the DNA damage response in human embryonic stem cells. Stem Cells. 2012;30(7):1362-72.

47. Faraonio R, Salerno P, Passaro F, Sedia C, laccio A, Bellelli R, et al. A set of miRNAs participates in the cellular senescence program in human diploid fibroblasts. Cell Death Differ. 2012;19(4):713-21.

48. Noll JE, Williams SA, Tong CM, Wang H, Quach JM, Purton LE, et al. Myeloma plasma cells alter the bone marrow microenvironment by stimulating the proliferation of mesenchymal stromal cells. Haematologica. 2014;99(1):163-71

49. Kuilman T, Michaloglou C, Mooi WJ, Peeper DS. The essence of senescence. Genes Dev. 2010;24(22):2463-79.

50. Manodoro F, Marzec J, Chaplin T, Miraki-Moud F, Moravcsik E, Jovanovic JV et al. Loss of imprinting at the $14 \mathrm{q} 32$ domain is associated with microRNA overexpression in acute promyelocytic leukemia. Blood. 2014;123(13):2066-74

51. Di Martino MT, Gulla A, Cantafio ME, Lionetti M, Leone E, Amodio N, et al. In vitro and in vivo anti-tumor activity of miR-221/222 inhibitors in multiple myeloma. Oncotarget. 2013;4(2):242-55.

\section{Submit your next manuscript to BioMed Central and take full advantage of:}

- Convenient online submission

- Thorough peer review

- No space constraints or color figure charges

- Immediate publication on acceptance

- Inclusion in PubMed, CAS, Scopus and Google Scholar

- Research which is freely available for redistribution 